'Departamento de Educación en Ciencias de la Salud. Facultad de Medicina. Universidad de Chile. Santiago Chile.

${ }^{2}$ Escuela de Enfermería. Facultad de Medicina. Universidad de Chile. Santiago Chile. ${ }^{3}$ Escuela de Fonoaudiología. Facultad de Medicina. Universidad de Chile. ${ }^{4}$ Escuela de Kinesiología. Facultad de Medicina. Universidad de Chile. ${ }^{5}$ Escuela de Obstreticia y Puericultura. Facultad de Medicina. Universidad de Chile. ${ }^{\mathrm{a}} \mathrm{PhD}$. bEnfermera universitaria. 'Fonoaudiólogo. 'Kinesióloga. eMatrona.

Recibido el 18 de diciembre de 2013, aceptado el 23 de junio de 2014.

Correspondencia a: Dr. Manuel Castillo Niño mcastillo@med.uchile.cl

\section{Cambio educativo en las Facultades de Medicina}

\author{
MANUEL CASTILLO ${ }^{1, a}$, GUSTAVO HAWES ${ }^{1}$, \\ SILVANA CASTILLO ${ }^{2, b}$, LUIS ROMERO ${ }^{3, c}$, ANA MARÍA ROJAS ${ }^{4, a}$, \\ MÓNICA ESPINOZA ${ }^{5, a}$, SANDRA OYARZO ${ }^{5, a}$
}

\section{The educational change in medical schools}

This paper reports the reflections of a group of members of the University of Chile Faculty of Medicine, about the changes in teaching methods that medical schools should incorporate. In a complex scenario, not only new and better knowledge should be transmitted to students but also values, principles, critical reasoning and leadership, among others. In the first part, a proposal to understand this educational development in the context of complex universities, incorporating pedagogical skills and reviewing institutional leadership, is carried out. In the second part, the training of teaching physicians, as part of the changes, is extensively discussed. Physicians hired as academics in the University should have the opportunity to work mainly as teachers and be relieved of research obligations. For them, teaching should become a legitimate area of academic development.

(Rev Med Chile 2014; 142: 1056-1060)

Key words: Curriculum; Education, pre medical; Faculty.
$\mathrm{E}$ n medicina es reconocido que la incorporación constante de nuevas tecnologías e innovaciones científicas traen avances para el abordaje de los procesos de salud enfermedad de las personas. Del mismo modo, los cambios en el diseño curricular y las prácticas de enseñanza, pueden convertirse en una oportunidad para proporcionar a los estudiantes sistemas de aprendizaje y planes de estudios más eficaces. Sin embargo, la implementación de cambios educativos asociados a estas innovaciones, es a menudo un desafío complejo para médicos y otros profesionales de la salud dedicados a la docencia y la administración de las escuelas universitarias, que se esfuerzan por lograr mayor calidad y pertinencia en sus programas formativos.

En la actualidad, desde diferentes sectores se demandan cambios en la formación de las carreras de la salud; el Gobierno le pide a las universidades que sus egresados estén preparados para enfrentar los cambios demográficos y sanitarios con altos niveles de calidad; los estudiantes reclaman no solamente gratuidad, sino que calidad y com- promiso social de las instituciones educativas; los académicos ven en sus respectivas disciplinas un permanente flujo de información a velocidades inimaginables ${ }^{1}$, que demandan constantes actualizaciones. Por su parte, desde las ciencias de la educación se responde intentando dejar de lado los aprendizajes memorísticos, poniendo énfasis en el desarrollo de la inteligencia y otras capacidades meta cognitivas como mecanismos para enfrentarse a las impredecibles exigencias profesionales futuras. En síntesis, las carreras de la salud se encuentran sometidas a demandas de cambios que provienen de fuentes externas e internas a las universidades y que las obligan a una constante revisión de sus estructuras y prácticas académicas.

En el contexto antes descrito, la literatura evidencia la necesidad de desarrollar cambios en la educación médica para dar respuesta a los desafíos asociados a la salud de las personas salud de las personas, adaptando a la comunidad universitaria a estos cambios y conducir programas rigurosos ${ }^{2}$, que busquen coherencia entre las necesidades 
sociales de salud y los planes de formación profesional desarrollados.

Lo anterior sin duda, revela la importancia de analizar el cambio educativo y los procesos asociados a la formación de los docentes como parte fundamental de dichos cambios; cuestión que constituye el tema central del presente artículo.

Este tema es abordado en numerosas publicaciones de revistas de especialidad de educación, educación médica y de educación en ciencias de la salud, analizando cambios e innovaciones curriculares, nuevas metodologías de enseñanza y aprendizaje, e instrumentos evaluativos; no obstante, son escasos los documentos que revisan el cambio educativo desde una dimensión social de la gestión organizacional más amplia.

A nuestro juicio, es necesaria una mirada sistémica que colabore a entender cómo funcionan estos cambios en las universidades, especialmente en aquellas llamadas complejas ${ }^{3}$. Para esto, nos acomoda el concepto de desarrollo educativo utilizado por Lynn para referirnos al conjunto de cambios que se están produciendo en las facultades de medicina ${ }^{4}$. De esta manera el propósito de este artículo es revisar los factores que influyen en el cambio educativo desde una visión amplia en las facultades de medicina y escuelas de la salud que corresponden a un modelo de universidades complejas.

Se presentan en primera instancia elementos centrales para el análisis del contexto vinculado a los cambios educativos, y posteriormente, se aborda la formación docente del médico y profesionales de la salud, como estrategia fundamental en los procesos de cambio.

\section{El contexto del cambio educativo}

El cambio en la enseñanza es ciertamente el factor final y decisivo que condiciona el éxito de los procesos de innovación y mejoramiento en la educación. Sin embargo, ni la innovación ni el desarrollo docente pueden concebirse fuera de los contextos organizacionales en que se dan. Según describen Stratton y otros colaboradores, los cambios curriculares expresan las experiencias educacionales las historias, misión y cultura local ${ }^{5}$. Tanto los componentes del medio externo como interno de la universidad tienen impacto sobre la innovación y, por consiguiente, sobre el desarrollo docente. Ronald Harden también menciona el contexto como un factor relevante en un análisis acerca del futuro de la educación médica ${ }^{6}$.

Como lo describen Robins y otros autores ${ }^{7}$, la educación médica se enfrenta con diferentes formas de estructura y organización curricular; por una parte facultades de estructuras de gobierno altamente verticales, lo que aparentemente puede facilitar los procesos de cambio educativo, $y$ aunque estos modelos ofrecen ventajas sobre las estructuras horizontales plantean otros desafíos para asegurar la calidad del plan de estudios y las innovaciones que impulsan. En este artículo se aborda el proceso de cambio en facultades de estructuras organizacionales horizontales que en general corresponden, en el caso chileno a universidades complejas, donde las universidades y sus académicos combinan las funciones de investigación, docencia y extensión. Además la estructura de gobierno es más democrática y la toma de decisiones es compartida, por lo tanto, los cambios educativos se enfrentan a contextos y procesos de mayor dificultad.

Considerando de manera prioritaria las variables internas, se encuentran condicionantes que residen en los aspectos propiamente organizacionales así como en lo cultural. Según Pheysey los factores organizacionales son aquellos que se relacionan: a) con el poder y las tensiones entre grupos de interés al interior de la institución educativa; b) las rutinas y las normativas que se orientan más a la conservación que al cambio; c) la necesaria coexistencia de modalidades de gestión -una para la conservación y otra para el cambioque no siempre es apacible; d) la disponibilidad y disposición de los recursos de todo tipo ${ }^{8}$.

Entre los factores culturales pueden señalarse de manera prioritaria aquellos relacionados con: a) la matriz ideológica sobre la que se sustenta la visión y misión de la institución así como la comprensión y apropiación que de la misma tengan los docentes; b) los hábitos culturales relacionados con el cambio y la significación de éste como "pérdida" o "ganancia"; c) la concepción fraccionaria del saber y la limitación de los intereses de los practicantes; d) la tensión entre los mundos del saber académico y el saber profesional ${ }^{9,10}$.

Todo esto pone de relieve la tensión entre el conservadurismo propio de las organizaciones educativas y las demandas de cambio. 
Los cambios educativos y la formación de los docentes

La formación de los médicos y otros profesionales de la salud en docencia es siempre parte de la estrategia de innovación. Las nuevas demandas de salud de la población han puesto de manifiesto la complejidad de las nuevas tareas docentes, la urgencia de abordar la especificidad del trabajo del profesional universitario, y la necesidad de establecer adecuados procesos formativos para estos docentes ${ }^{11}$. Los autores que investigan estos procesos de formación coinciden en afirmar que el desarrollo profesional docente ${ }^{12}$ es un concepto multidimensional entendido como un proceso amplio, flexible, continuo, y caracterizado por una reflexión constante sobre la experiencia cotidiana. El quehacer diario de los médicos incluye oportunidades y experiencias que promueven el crecimiento y el desarrollo en la profesión docente. Carlos Marcelo reconoce este desarrollo como proceso de construcción de una nueva cultura profesional, se concibe como un proceso colaborativo, que sin suprimir espacio para el trabajo y la reflexión personal, consigue los mayores beneficios en las interacciones significativas con otros docentes y, por tanto, implica la consideración del médico-docente como aprendiz activo y comprometido en la preparación, observación y reflexión sobre su práctica docente ${ }^{13}$.

Ahora bien, Lobato y otros autores ${ }^{14,15}$ señalan que para la generación de innovaciones y la implementación del cambio educativo en la facultades de medicina es necesario integrar equipos de trabajo eficientes con quienes son capaces de comprometerse en las tareas y con el cambio institucional ${ }^{16}$. En estos equipos docentes la discusión permite construir conocimiento pedagógico, un conocimiento contextualizado que emerge desde la interrelación entre los docentes que participan. En este sentido Gairín alude a la importancia de ir consolidando una cultura colaborativa en las facultades de medicina que genera, entre otros efectos, la potenciación y el desarrollo profesional de su capital humano ${ }^{17}$.

Parece evidente que el cambio educativo en las facultades de medicina nos conduce a un modo muy distinto de entender el trabajo como docentes $^{18,17}$. Una revisión relevante de este tema ${ }^{19,20}$ subraya el impacto que los cambios están teniendo en la identidad del médico docente, hasta el punto de tener que hablar de "identidades en transición" debido a la dificultad de establecer un perfil docente ideal $o$ al que podamos aspirar para dar respuesta a los cambios actuales.

En lo que al parecer no hay dudas, es cómo influyen los docentes en la calidad de los sistemas educativos, incluidas las carreras de la salud. Al respecto Barber en el informe "Cómo hicieron los sistemas educativos con mejor desempeño en el mundo para alcanzar sus objetivos" del año 2008, señala que la calidad de un sistema educativo tiene como techo la calidad de sus docentes ${ }^{21}$. Después de estudiar veinticinco sistemas educativos de todo el mundo, incluidos los diez sistemas con mejor desempeño, y analizando qué tienen en común estos sistemas con alto desempeño y cuáles son las herramientas que emplean para mejorar los resultados de sus estudiantes, resaltan la importancia de conseguir a las personas más aptas para ejercer la docencia y formarlas hasta convertirlos en docentes eficaces.

Los protagonistas del cambio siempre son los docentes a través de sus formas de interactuar con los estudiantes, por lo que una innovación sólo se adoptará cuando los docentes conozcan, compartan e incorporen en profundidad la innovación propuesta. Las razones que fundamenten la necesidad de formar a los docentes en los procesos de cambio parecen obvias, pero al analizar las necesidades percibidas por los docentes para cambiar no siempre son las mismas. Un estudio reciente de Espinoza en la Escuela de Obstetricia de la Universidad de Chile, muestra que los académicos con más experiencia docente manifiestan la necesidad de conocer los fundamentos teóricos del cambio para involucrarse en los procesos de modificación de sus prácticas, mientras que aquellos con menor experiencia docente demandan principalmente capacitación en las nuevas metodologías que las innovaciones requieren ${ }^{22}$. Cualquiera de las situaciones mencionadas y otras implican procesos formativos para los docentes participantes.

Por parte de quienes dirigen los procesos de formación de docentes es frecuente creer que una o dos sesiones de formación inicial presentando el nuevo modelo son "suficientes". La experiencia de los autores de este artículo en programas de formación de docentes en la Facultad de Medicina de la Universidad de Chile nos indica que la formación continua y de profundidad es fundamental ${ }^{23}$.

Esta preocupación por la calidad de la for- 
mación ha llevado también a preguntarse por la efectividad de los métodos empleados en ella, Joyce y Showers explican que el seguimiento, junto con la oportunidad de participar en la resolución de problemas relacionados con las nuevas metodologías otros con colegas, puede mejorar significativamente la implementación ${ }^{23}$. De hecho, Fullan menciona que es después de tratar de aplicar nuevas prácticas educativas con resultados frustrantes, es que los docentes sienten más la necesidad de apoyo adicional y formación ${ }^{24}$.

Pareciera que la utilización de diversos métodos de formación tales como cursos o talleres, seguimientos, asesorías y acompañamientos según las circunstancias de cada proceso de cambio, son claves para el éxito. También es necesario indicar que a nivel nacional Triviño y cols han publicado resultados similares en la Universidad Católica al respecto ${ }^{25}$.

Otra práctica de formación es la prestación de apoyo y desarrollo de habilidades para los líderes pedagógicos, tales como directores, coordinadores de niveles, encargados de cursos y otros. Como afirma Murillo, en estos actores es especialmente importante la formación en procesos de gestión del cambio, que les permita avanzar con los conflictos de resistencias que naturalmente emergen durante la implantación de las innovaciones ${ }^{26}$. Debido a que el proceso de cambio es largo y exigente, nuevos líderes deben ser contratados para hacerse cargo cuando los actuales necesiten descanso y renovación.

Lynn y otros afirman que cuando nuevos miembros se unen a la facultad durante el proceso de cambio, es importante que sean socializados en la innovación. Aun cuando estos nuevos miembros apoyen la iniciativa de cambio deben ser puestos al tanto en la forma en que el proceso de implementación en particular está ocurriendo. Los nuevos miembros pueden alterar involuntariamente un nuevo programa frágil porque no están suficientemente familiarizados con la cultura de la organización o contexto ${ }^{4}$.

Así como la atención especial se debe tener con los nuevos miembros, incluso consultores expertos si se emplean, deben utilizarse juiciosamente. El personal no debe llegar a ser demasiado dependiente de consultores externos. Por el contrario, los miembros de la organización deben estar capacitados para asumir la plena responsabilidad del proyecto para asegurar su continuidad en el tiempo ${ }^{4}$. La madurez de la institución se verifica cuando es posible gestionar estos procesos de cambio de forma autónoma, es decir, cuando directivos y docentes han incorporado a sus tareas las nuevas concepciones y prácticas.

Ofrecer recompensas por la participación en los cambios curriculares en las escuelas de medicina es particularmente difícil. Nos acomoda la propuesta de Kanter que sugiere el diseño y la promoción de un programa innovador como un experimento. Este término eleva la innovación en los ojos de los profesores y administradores y pasa de ser una tarea de enseñanza a ser un estudio de investigación, merecedora de la organización en inversión de esfuerzo. Los experimentos se toman en serio en la comunidad científica ${ }^{27}$, especialmente en las facultades de medicina y de la salud.

En síntesis, las facultades de medicina en Chile especialmente aquellas con estructuras complejas, han asumido que los cambios educativos tienen algunos aspectos visibles y relevantes, como son las prácticas docentes según lo describen varios autores como Zabalza, Triviño y otros ${ }^{12}$, y adicionalmente, coincidiendo con lo planteado por Fullan $^{28}$, han incorporado también un análisis de los cambios desde la dimensión social, la cultura, la comunicación y el liderazgo. Esto constituye buenas noticias, considerando que Harden ${ }^{6}$ indica que el futuro de la educación médica se encuentra en un proceso de cambio en distintos ámbitos; enfoques interconectados internacionales, importante movilidad de estudiantes, docentes y currículos a través de las fronteras de los países. Desde esta mirada el estudio de las carreras de la salud está siendo exigido para cambiar desde diversos ángulos y para dar cuenta de una realidad cada vez más compleja, y es aquí donde la comprensión de estos procesos se vuelve estratégica.

\section{Referencias}

1. Herskovic P, La Reforma Curricular en la Escuela de Medicina de la Universidad de Chile. Rev Chil Pediatr 2005; 76 (1): 9-11.

2. Steinert Y, Mann K, Centeno A, Dolmans D, Spencer J, Gelula $\mathrm{M}$, et al. A systematic review of faculty development initiatives designed to improve teaching effectiveness in medical education. Medical teacher, 2006. BEME Guide $N^{\circ} 8.28$ (6): 497-526.

3. Parada J. Universidades Públicas y Privadas. Un enfoque 
tridimensional. Estudios Públicos, 2010. (120): p. 183 205.

4. Lynn K, Rege N. Making the shift from Faculty development to educational development, in: Building Teaching Capacities in Higher Education: A Comprehensive International Model, A Saroyan and M Frenay, Editors Virginia, USA: Stylus Publishing 2010.

5. Stratton T, Rudy D, Sauer M, Perman M, Jennings J. Lessons from Industry: One School's. Transformation Toward "Lean" Curricular Governance. Acad Med 2007 82 (331): p. 331-40.

6. Harden RM. International medical education and future directions: a global perspective. Acad Med 200681 (12): p. S22-S29.

7. Robins L, White C, Fantone J. The difficulty of sustaining curricular reforms. A study of "Drift" at one school. Acad Med 2000 (75 8): p. 801-5.

8. Pheysey DC. Organizational Cultures. Types and Transformations. London, UK: Routledge; 1993.

9. Trowler P. Academics Responding to change. New Higher Education Frameworks and Academic Cultures. London, UK: Society for Research into Higher Education. 1998.

10. Gornitzka A, Kogan M, Amaral A. Reform and Change in Higher Education. Analysing Policy Implementation 2005, Netherlands Springer.

11. Fullan RK. Team-centered discharge management: on a fast track to performance improvement. Continuum, 1995. 15 (3): p. 1, 3-9.

12. Zabalza M. Competencias Docentes del Profesorado Universitario. N. ediciones. 2007, Madrid: Narcea ediciones.

13. Marcelo C. Pensamientos pedagógicos y toma de decisiones: Un estudio sobre la planificación de la enseñanza y decisiones didácticas de los profesores de E.G.B., Conocimiento, Creencias y Teorías de los Profesores. UK: Marfil Editor. 2008: p. 277-301.

14. Lobato C, Fernández I, Garmendia M, Pérez U. ¿Se puede construir la identidad del profesorado en la universidad? Una experiencia desde la formación del profesorado novel en la Universidad del País Vasco. CIDUI 2012 - Llibre d'actes, España: Universidad del País Vasco; 2012.

15. Nemiña E, García H, Montero L. Desarrollo profesional y profesionalización docente. Perspectivas y problemas. Profesorado: Revista de currículum y formación del profesorado, 2009. 13 (2): 1-13.

16. Tardif M. Los saberes del docente y su desarrollo profesional. Madrid España: Narcea. 2004.

17. Gairín J. Cambio de cultura y organizaciones que aprenden. Educar 2000; 27: 31-85.

18. Banchero P, Castillo M. ¿Cómo se construye la identidad docente en Medicina? 2008, Santiago-Chile. Universidad de Chile.

19. Buck B. Reformar la FP hacia la formación permanente: retos para la docencia. Revista Europea de Formación Profesional 2005; 35: 23-35.

20. De Laat M, Simons RJ. El aprendizaje colectivo: perspectivas teóricas y modelos que apoyan la formación coordinada. Revista Europea de Formación Profesional, 2002; 27: 14-27.

21. Baber M, Mourshed M. Cómo hicieron los sistemas educativos con mejor desempeño del mundo para alcanzar sus objetivos. 2008, PREAL: McKinsey \& Company.

22. Espinoza M. El saber pedagógico de los docentes de la Escuela de Obstetricia de la Universidad de Chile y los procesos de cambio curricular. Tesis de Magíster en Educación en Ciencias de la Salud. Departamento de Educación en Ciencias de la Salud. Universidad de Chile: Facultad de Medicina. 2013.

23. Castillo M. Qué creen de la docencia los académicos de las carreras de la salud en la Facultad de Medicina de la Universidad de Chile. Revista de Docencia Universitaria REDU 2012; 10: 243-56.

24. Joyce B. Showers B. Student Achievement through Staff Development, in Designing Training and PeerCoaching: Our needs for learning. VA. USA: ASCD Editor; 2002.

25. Triviño X, Sirhan M, Moore P, Reyes C. Formación en educación de los docentes clínicos de medicina. Revista Med Chile 2011; 137 (11): 1516-22.

26. Murillo-Estepa P, Mayor C, Hernández E, Sánchez M. Las necesidades formativas docentes de los profesores universitarios. Revista de la Facultad de Ciencias de la Educación. 2004 (6): 97-116.

27. Kanter R. The Change Masters: Innovation for Productivity in the American Corporation. New York: Simon \& Shuster. 1983.

28. Fullan M. The future of educational change: system thinkers in action. Journal of Educational Change, 2006 (7): 113-22. 